\title{
Recurrent Clostridium difficile-Associated Diarrhea and Colitis Treated with Saccharomyces cerevisiae (Baker's Yeast) in Combination with Antibiotic Therapy: A Case Report
}

\author{
Donald J. Kovacs, MD, and Ted Berk, MD, FACP
}

Antibiotic-associated diarrhea is common in both inpatient and outpatient settings, and $15 \%$ to $20 \%$ of the cases are caused by Clostridium difficile. ${ }^{1} \mathrm{Al}-$ though antibiotic therapy with metronidazole or other agents is effective for most patients with $C$ difficile-associated diarrhea, approximately $10 \%$ to $20 \%$ of patients receiving treatment for $C$ difficile infection will experience a relapse following discontinuation of initial therapy, ${ }^{2}$ and relapses are often multiple. This clinical problem can be frequent and frustrating.

$C$ difficile-associated diarrhea is thought to occur when an alteration of the normal bacterial flora of the intestinal tract permits proliferation of toxigenic strains of $C$ difficile. Various microorganisms have been used therapeutically to repopulate the intestinal flora to enhance resistance against $C$ difficile. A randomized placebo-controlled trial has shown that the yeast Saccbaromyces boulardii is effective for the treatment of recurrent $C$ difficile disease. ${ }^{3}$ This biotherapeutic agent, however, is not available as a pharmaceutical preparation in the United States. We report herein a case of $C$ difficileassociated diarrhea with multiple relapses that responded to treatment with antibiotics together with a commercial baker's yeast (Fleischmann's Yeast), which contains the closely related species Saccharomyces cerevisiae at a concentration of organisms at $10^{10} / \mathrm{g}$.

\section{Case Report}

A 50-year-old woman came to the office complaining of a 1-week history of diarrhea and weakness with crampy lower abdominal pain and 4 days of

Submitted, revised, 10 December 1999.

From the Carlisle Hospital (DJK, TB), Carlisle, Penna. Address reprint requests to Donald J. Kovacs, MD, Yellow Breeches Family Practice, 1358 Lutztown Road, Boiling Springs, PA 17007. vomiting and low-grade fever. One month earlier she had undergone a laparotomy with hysterectomy and bilateral salpingo-oophorectomy for drainage of a large pelvic abscess. She had received intravenous antibiotics during her hospitalization and was sent home from the hospital with a prescription for oral clindamycin, $300 \mathrm{mg}$ four times a day, which she had taken for 20 days.

The patient was given a prescription for oral metronidazole for a presumptive diagnosis of antibiotic-associated colitis, but she required hospitalization the following day because of intractable vomiting and abdominal pain. A stool sample was guaiac negative, but it was positive for $C$ difficile by enzyme-linked fluorescent immunoassay (ELFA) performed with the automated VIDAS instrument and CDA Dual Reagent Strips (bioMérieux Vitek, Inc., Hazelwood, Mo). Her white blood cell count was $7300 / \mu \mathrm{L}$. A bowel obstruction series was negative, and a computed tomographic scan of the abdomen and pelvis showed no evidence of recurrent abscess. She continued to vomit when given oral metronidazole, so she was given intravenous metronidazole and oral vancomycin for 4 days. She was released from the hospital with instructions to continue taking vancomycin, $125 \mathrm{mg}$ four times a day, for 5 more days.

Several days after discharge she developed hives, and the vancomycin was stopped. A few days later she developed recurrent diarrhea. Oral metronidazole was started, but she developed nausea, vomiting, and abdominal cramping. The metronidazole was stopped on the suspicion that these symptoms might be side effects, and cholestyramine was prescribed. After a day or 2 she developed severe abdominal cramping, and the cholestyramine was stopped. The vancomycin was restarted without recurrent hives, but she continued to have nausea, vomiting, and diarrhea, and she was readmitted to the hospital. She was given intravenous metronida- 
zole for 6 days. Her $C$ difficile toxin assay was again positive, and a sigmoidoscopic examination showed classic-appearing pseudomembranes. After she was sent home from the hospital, she continued taking oral vancomycin for about 2 weeks.

One week after stopping the vancomycin she noted recurrence of nausea and the following day developed diarrhea. The vancomycin was started again, but because of the vomiting, she was admitted for the third time for colitis. Treatment was started with intravenous metronidazole, and she responded quickly. She was released from the hospital with a prescription for vancomycin, $125 \mathrm{mg}$ four times a day for 10 days, then twice a day for 7 days, then every other day for 3 weeks, along with a lactobacilli preparation (Lactinex) $1 \mathrm{~g}$ four times a day for 3 weeks.

Two weeks later the patient was readmitted a fourth time, just 2 days after switching to the twicea-day regimen, with vomiting and abdominal discomfort. Although she had not yet developed diarrhea, she believed her symptoms were the same as with previous relapses. Findings of a repeat sigmoidoscopy to $30 \mathrm{~cm}$ were normal. An infectious disease consultant recommended treatment with Saccharomyces cerevisiae (Fleischmann's Yeast) 1/8 tsp, four times a day for 4 months, and vancomycin $125 \mathrm{mg}$ four times a day for 30 days. With this course of treatment there have been no further recurrences in more than 3 years.

\section{Discussion}

$C$ difficile is a spore-forming obligate anaerobic bacillus and is a component of normal fecal flora in about $5 \%$ of healthy adults. It can be found in the stool of $10 \%$ or more of hospitalized adults without diarrhea who have received antibiotics or chemotherapeutic agents. In $C$ difficile diarrhea, colitis both with and without pseudomembranes is due to toxin-mediated mucosal inflammation. The most commonly implicated antibiotics are ampicillin and other penicillin derivatives, cephalosporins, and clindamycin.

The typical clinical manifestations of $C$ difficile diarrhea are crampy abdominal pain, profuse diarrhea, low-grade fever, and leukocytosis. The onset can be as soon as after a few days of antibiotic therapy, or it might be delayed for up to 8 weeks after its discontinuation. Fever can be as high as $40^{\circ} \mathrm{C}$, and leukocytosis can reach $50,000 / \mu \mathrm{L}$. $^{2}$ The colitis is usually most severe in the distal colon and rectum, but it can occur throughout the colon. Colitis localized to the cecum might cause little or no diarrhea, but it can cause fever, marked right lower quadrant abdominal pain and tenderness, marked leukocytosis, and decreased intestinal motility. ${ }^{4}$

Endoscopy with biopsy of suspect lesions is the most rapid way to establish the diagnosis of $C$ difficile colitis. The characteristic lesions are raised, yellowish nodules or plaque-like pseudomembranes, often with skip areas of normal mucosa. Typical pseudomembranes are often absent, however. Tissue culture tests for toxin $B$ are the reference standard and can detect the specific cytopathic effects of toxin B in more than $90 \%$ of patients with pseudomembranous colitis. A negative test, however, does not rule out $C$ difficile as the cause of diarrhea. It is also not uncommon for patients to continue to have positive tests for toxin $B$ or the organism after otherwise successful therapy. Enzyme immunoassay tests for toxin $A$ or $B$ are the most widely used tests to diagnose $C$ difficile infection. They are more specific than they are sensirive, with sensitivities ranging from $70 \%$ to $95 \%$. The sensitivity can be improved by sending a second stool sample the next day, rather than the same day. ${ }^{1}$ Stool cultures for $C$ difficile can yield falsepositive results in patients with simple antibioticinduced diarrhea who have coincidental colonization with $C$ difficile. The finding of fecal neutrophils raises the suspicion of $C$ difficile-associated diarrhea.

Treatment of $C$ difficile-associated diarrhea includes supportive therapy with replacement of fluids and electrolytes. The patient should stop taking antibiotics, if possible. Treatment with antiperistaltic or opiate antidiarrheal agents should be avoided. Metronidazole, preferably given orally, is the drug of choice for treating $C$ difficile-associated diarrhea. The usual dose of oral metronidazole is $250 \mathrm{mg}$ four times a day for 10 days.

Vancomycin was prescribed for the patient reported above because she was unable to tolerate oral metronidazole. Vancomycin is to be discouraged, however, so as not to promote the spread of vancomycin-resistant enterococci and staphylococci. ${ }^{5}$ Vancomycin is also much more expensive and proved no more effective than metronidazole in a prospective randomized trial comparing the two agents. ${ }^{6}$ 
Relapses or recurrences, defined as the return of symptoms, signs, and positive diagnostic tests a few weeks to months after discontinuing successful treatment of $C$ difficile diarrhea, are common, and occur in $15 \%$ to $35 \%$ of patients. ${ }^{1}$ As in this case, relapses usually respond well to treatment, but multiple recurrences are common, and up to 20 have been reported in a single patient.

Metronidazole and vancomycin do not reliably kill the spore forms of $C$ difficile; in fact, they might encourage the formation of spores. The persistence of both the spores and the antibiotic-induced reduction of the colonic bacterial flora presumably account for the propensity for relapse.

There is no specific regimen that has been clearly proved to prevent recurrences. Longer courses of metronidazole or vancomycin therapy have been tried, sometimes with every-other-day administration or gradual tapering. Lactobacillus preparations or yogurt with live cultures are sometimes tried. Two small open trials involving a total of 9 patients have suggested that Lactobacillus casei GG might be effective for the treatment of recurrent $C$ difficile colitis. ${ }^{7,8}$

The decision to use yeast to treat $C$ difficileassociated diarrhea in our patient was based on a promising randomized, double-blind, controlled study using live yeast (Saccharomyces boulardii) to treat $C$ difficile-related diarrhea. ${ }^{3}$ In that study, the addition of $S$ boulardii to standard antibiotic treatment in 60 patients with recurrent $C$ difficile disease reduced the subsequent relapse rate by about $50 \%$ when compared with placebo plus antibiotics. In 64 patients with $C$ difficile disease initially, there was no significant difference in benefit with the addition of $S$ boulardii, although because so few patients with initial disease failed, there was only a $10 \%$ power of detecting a significant difference. Patients who were immunocompromised as a result of AIDS or cancer chemotherapy were excluded from the study. The two adverse reactions noted with $S$ boulardii were increased thirst and constipation. Because $S$ boulardii is not readily available in the United States, some physicians have tried $S$ cerevisiae (baker's yeast, found in Fleishmann's Yeast) with anecdotal success. ${ }^{9}$ The principal author of the $S$ boulardii study, however, responded to this report with the assertion that $S$ cerevisiae is a completely different species and cited controlled experiments in mice where $S$ boulardii showed a signifi- cant protective effect against $C$ difficile whereas $S$ cerevisiae did not. ${ }^{10}$

\section{Conclusion}

A case of well-documented $C$ difficile diarrhea with four recurrences is described in which a novel and unproved variation of a yeast-based therapy given with a more prolonged course of oral vancomycin was followed by no further recurrences. Whereas no conclusions can be made about the efficacy of $S$ cerevisiae for preventing recurrences of $C$ difficile diarrhea, this case left a strong impression. Further study of this inexpensive and readily available treatment should be considered.

Ernest Davis, DO, the infectious disease consultant, recommended the use of baker's yeast in this case.

\section{References}

1. Fekety R. Guidelines for the diagnosis and management of Clostridium-difficile-associated diarrhea and colitis. American College of Gastroenterology, Practice Parameters Committee. Am J Gastroenterol 1997;92:739-50.

2. Kelly CP, Pothoulakis C, LaMont JT. Clostridium difficile colitis. N Engl J Med 1994;330:257-62.

3. McFarland LV, Surawicz CM, Greenberg RN, et al. A randomized placebo-controlled trial of Saccharomyces boulardii in combination with standard antibiotics for Clostridium difficile disease. JAMA 1994;271: 1913-8.

4. Triadafilopoulos G, Hallstone AE. Acute abdomen as the first presentation of pseudomembranous colitis. Gastroenterology 1991;100:685-91.

5. Recommendations for preventing the spread of vancomycin resistance: recommendations of the Hospital Infection Control Advisory Committee (HICPAC). Am J Infect Control 1995;23:87-94.

6. Teasley DG, Gerding DN, Olson MN, et al. Prospective randomized trial of metronidazole versus vancomycin for Clostridium-difficile-associated diarrhoea and colitis. Lancet 1983;2:1043-6.

7. Gorbach SL, Chang TW, Goldin B. Successful treatment of relapsing Clostridium difficile colitis with Lactobacillus GG. Lancet 1987;2:1519.

8. Biller JA, Katz AJ, Flores AF, Buie TM, Gorbach SL. Treatment of recurrent Clostridium difficile colitis with Lactobacillus GG. J Pediatr Gastroenterol Nutr 1995;21:224-6.

9. Chia JK, Chan SM, Goldstein H. Baker's yeast as adjunctive therapy for relapses of Clostridium difficile diarthea. Clin Infect Dis 1995;20:1581.

10. McFarland LV. Saccharomyces boulardii is not Saccharomyces cerevisiae. Clin Infect Dis 1996;22:200-1. 